\title{
Breadth of concomitant immune responses prior to patient recovery: a case report of non-severe COVID-19
}

To the Editor - We report the kinetics of immune responses in relation to clinical and virological features of a patient with mild-to-moderate coronavirus disease 2019 (COVID-19) that required hospitalization. Increased antibody-secreting cells (ASCs), follicular helper T cells ( $\mathrm{T}_{\mathrm{FH}}$ cells), activated $\mathrm{CD}^{+} \mathrm{T}$ cells and $\mathrm{CD} 8^{+} \mathrm{T}$ cells and immunoglobulin $\mathrm{M}$ (IgM) and IgG antibodies that bound the COVID-19causing coronavirus SARS-CoV-2 were detected in blood before symptomatic recovery. These immunological changes persisted for at least $7 \mathrm{~d}$ following full resolution of symptoms.

A 47-year-old woman from Wuhan, Hubei province, China, presented to an emergency department in Melbourne, Australia. Her symptoms commenced $4 \mathrm{~d}$ earlier with lethargy, sore throat, dry cough, pleuritic chest pain, mild dyspnea and subjective fevers (Fig. 1a). She traveled from Wuhan to Australia $11 \mathrm{~d}$ before presentation. She had no contact with the Huanan seafood market or with known COVID-19 cases. She was otherwise healthy and was a non-smoker taking no medications. Clinical examination revealed a temperature of $38.5^{\circ} \mathrm{C}$, a pulse rate of 120 beats per minute, a blood pressure of $140 / 80 \mathrm{~mm} \mathrm{Hg}$, a respiratory rate of 22 breaths per minute, and oxygen saturation 98\% while breathing ambient air. Lung auscultation revealed bi-basal rhonchi. At presentation on day 4, SARS-CoV-2 was detected in a nasopharyngeal swab specimen by real-time reverse-transcriptase PCR. SARS-CoV- 2 was again detected at days 5-6 in nasopharyngeal, sputum and fecal samples, but was undetectable from day 7 (Fig. 1a). Blood C-reactive protein was elevated at 83.2, with normal counts of lymphocytes $\left(4.3 \times 10^{9}\right.$ cells per liter (range, $4.0 \times 10^{9}$ to $12.0 \times 10^{9}$ cells per liter)) and neutrophils $\left(6.3 \times 10^{9}\right.$ cells per liter (range, $2.0 \times 10^{9}$ to $8.0 \times 10^{9} \times 10^{9}$ cells per liter)). No other respiratory pathogens were detected. Her management was intravenous fluid rehydration without supplemental oxygenation. No antibiotics, steroids or antiviral agents were administered. Chest radiography demonstrated bi-basal infiltrates at day 5 that cleared on day 10 (Fig. 1b). She was discharged to home isolation on day 11. Her symptoms resolved completely by day 13 , and she remained well at day 20, with progressive increases in plasma SARS-CoV-2-binding IgM and IgG antibodies from day 7 until day 20 (Fig. 1c and Extended Data Fig. 1). The patient was enrolled through the Sentinel Travelers Research Preparedness Platform for Emerging Infectious Diseases novel coronavirus substudy (SETREP-ID-coV) and provided written informed consent before the study. Patient care and research were conducted in compliance with the Case Report guidelines and the Declaration of Helsinki. Experiments were performed with ethics approvals HREC/17/MH/53, HREC/15/MonH/64/2016.196 and UoM\#1442952.1/\#1443389.4.

We analyzed the kinetics and breadth of immune responses associated with clinical resolution of COVID-19. As ASCs are key for the rapid production of antibodies following infection with Ebola virus ${ }^{1,2}$ and infection with and vaccination against influenza virus ${ }^{2,3}$, and activated circulating $\mathrm{T}_{\mathrm{FH}}$ cells $\left(\mathrm{cT}_{\mathrm{FH}}\right.$ cells) are concomitantly induced following vaccination against influenza virus ${ }^{3}$, we defined the frequency of $\mathrm{CD} 3{ }^{-} \mathrm{CD} 19^{+} \mathrm{CD} 27^{\mathrm{hi}} \mathrm{CD} 38^{\text {hi }}$ ASC and $\mathrm{CD}^{+}{ }^{+} \mathrm{CXCR}^{+} \mathrm{ICOS}^{+} \mathrm{PD}-1^{+} \mathrm{cT}_{\mathrm{FH}}$ cell responses before symptomatic recovery. ASCs appeared in the blood at the time of viral clearance (day $7 ; 1.48 \%$ ) and peaked on day $8(6.91 \%)$. The emergence of $\mathrm{cT}_{\mathrm{FH}}$ cells occurred concurrently in blood at day $7(1.98 \%)$, increasing on day $8(3.25 \%)$ and day 9 (4.46\%) (Fig. 1d). The peak of both ASCs and $\mathrm{cT}_{\mathrm{FH}}$ cells was markedly higher in the patient with COVID-19 than in healthy control participants $(0.61 \% \pm 0.40 \%$ and $1.83 \% \pm 0.77 \%$, respectively (average \pm s.d.); $n=5$ ). Both ASCs and $\mathrm{cT}_{\mathrm{FH}}$ cells were prominently present during convalescence (day 20) (4.54\% and $7.14 \%$, respectively; Fig. 1d). Thus, our study provides evidence on the recruitment of both ASCs and $\mathrm{cT}_{\mathrm{FH}}$ cells in this patient's blood while she was still unwell and $3 \mathrm{~d}$ before the resolution of symptoms.

Since co-expression of CD38 and HLA-DR is the key phenotype of the activation of $\mathrm{CD} 8^{+} \mathrm{T}$ cells in response to viral infections, we analyzed co-expression of CD38 and HLA-DR. As per reports for Ebola and influenza ${ }^{1,4}$, co-expression of CD38 and HLA-DR on
$\mathrm{CD}^{+} \mathrm{T}$ cells (assessed as the frequency of $\mathrm{CD}_{3} 8^{+} \mathrm{HLA}-\mathrm{DR}^{+} \mathrm{CD} 8^{+} \mathrm{T}$ cells) rapidly increased in this patient from day $7(3.57 \%)$ to day $8(5.32 \%)$ and day 9 (11.8\%), then decreased at day $20(7.05 \%)$ (Fig. 1e). Furthermore, the frequency of CD $38^{+}{ }^{H L A}-D^{+}{ }^{+} \mathrm{CD}^{+} \mathrm{T}$ cells was much higher in this patient than in healthy individuals $(1.47 \% \pm 0.50 \%$; $n=5)$. $\mathrm{CD}^{2} 8^{+} \mathrm{HLA}-\mathrm{DR}^{+} \mathrm{T}$ cells were also recently documented in a patient with COVID-19 at one time point ${ }^{5}$. Similarly, co-expression of CD38 and HLA-DR on $\mathrm{CD} 4^{+} \mathrm{T}$ cells (assessed as the frequency of CD $38^{+}{ }^{H L A}-\mathrm{DR}^{+} \mathrm{CD} 4^{+} \mathrm{T}$ cells) increased between day 7 (0.55\%) and day $9(3.33 \%)$ in this patient, relative to that of healthy donors $(0.63 \% \pm 0.28 \% ; n=5)$, although at lower levels than that of $\mathrm{CD} 8^{+} \mathrm{T}$ cells. CD $38^{+}{ }^{+} \mathrm{LA}-\mathrm{DR}^{+} \mathrm{T}$ cells, especially CD8 ${ }^{+}$ $\mathrm{T}$ cells, produced larger amounts of granzymes A and B and perforin ( $34-54 \%$ higher) than did their parent cells (CD8 ${ }^{+}$ or $\mathrm{CD}^{+}$populations; Fig. 1e). Thus, the emergence and rapid increase in activated CD $38^{+} \mathrm{HLA}_{-} \mathrm{DR}^{+} \mathrm{T}$ cells, especially $\mathrm{CD} 8^{+}$ $\mathrm{T}$ cells, at days 7-9 preceded the resolution of symptoms. Details on data reproducibility are in the Life Sciences Reporting Summary.

Analysis of $\mathrm{CD} 16^{+} \mathrm{CD} 14^{+}$monocytes, which are related to immunopathology, showed lower frequencies of $\mathrm{CD} 16^{+} \mathrm{CD} 14^{+}$ monocytes in the blood of this patient at days 7,8 and $9(1.29 \%, 0.43 \%$ and $1.47 \%$, respectively) than in that of healthy control donors (9.03\% $\pm 4.39 \%$; $n=5$ ) (Fig. 1f), possibly indicative of the efflux of $\mathrm{CD} 16^{+} \mathrm{CD} 14^{+}$monocytes from the blood to the site of infection. No differences in activated $\mathrm{HLA}-\mathrm{DR}^{+} \mathrm{CD} 3^{-} \mathrm{CD} 56^{+}$natural killer cells were found.

As pro-inflammatory cytokines and chemokines are predictive of severe clinical outcomes for influenza ${ }^{6}$, we quantified 17 pro-inflammatory cytokines and chemokines in plasma. We found low levels of the chemokine MCP-1 (CCL2) in the patient's plasma (Extended Data Fig. 2a), although this was comparable to results obtained for healthy donors $(22.15 \pm 13.81$; $n=5$ ), patients infected with influenza A virus or influenza $B$, assessed at days 7-9 $(33.85 \pm 30.12 ; n=5)$, and a patient infected with the human coronavirus HCoV-229e (40.56). Thus, in contrast to severe avian 
a
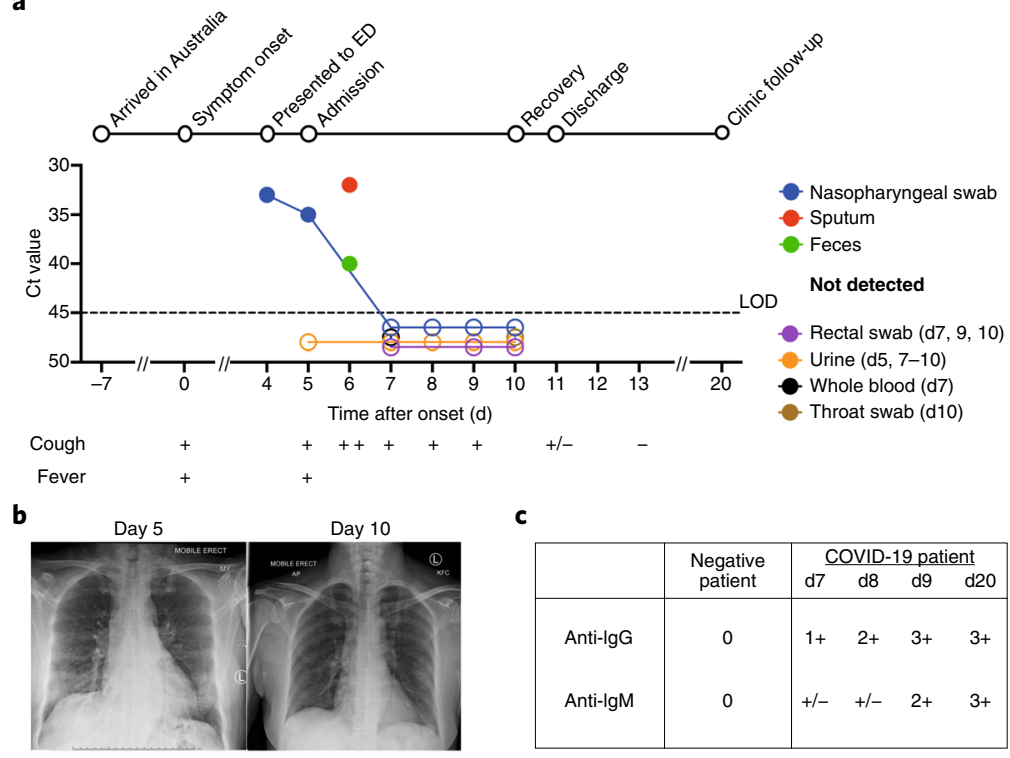

d

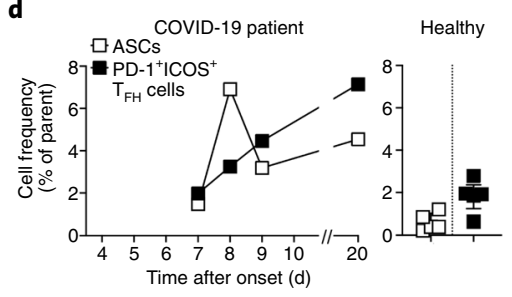

e

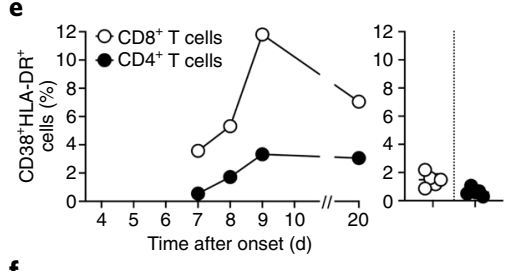

f

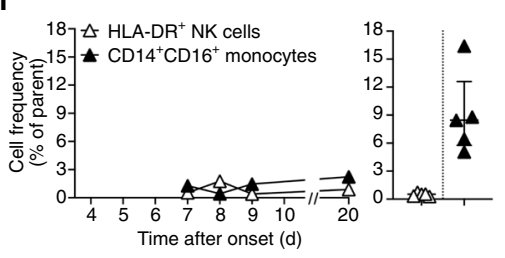

c

\begin{tabular}{|c|c|c|c|c|c|}
\hline & \multirow{2}{*}{$\begin{array}{c}\text { Negative } \\
\text { patient }\end{array}$} & \multicolumn{4}{|c|}{ COVID-19 patient } \\
\hline & & d7 & $\mathrm{d} 8$ & d9 & d20 \\
\hline Anti-lgG & 0 & $1+$ & $2+$ & $3+$ & $3+$ \\
\hline Anti-lgM & 0 & $+/-$ & +1 & $2+$ & $3+$ \\
\hline
\end{tabular}
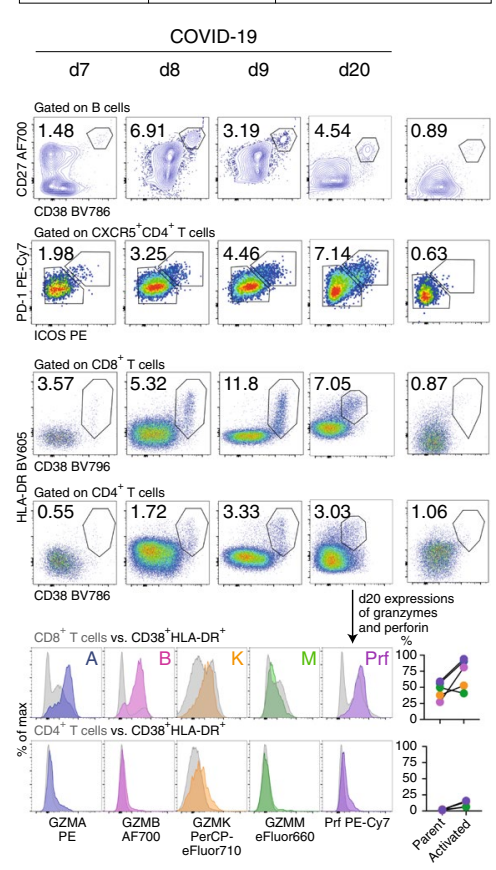

Fig. 1 | Emergence of immune responses during non-severe symptomatic COVID-19. a, Timeline of COVID-19, showing detection of SARS-CoV-2 in sputum, nasopharyngeal aspirates and feces but not urine, rectal swab or whole blood. SARS-CoV-2 was quantified by rRT-PCR; cycle threshold $(\mathrm{Ct})$ is shown. A higher $\mathrm{Ct}$ value means lower viral load. Dashed horizontal line indicates limit of detection (LOD) threshold $(\mathrm{Ct}=45)$. Open circles, undetectable SARS-CoV-2. b. Anteroposterior chest radiographs on days 5 and 10 following symptom onset, showing radiological improvement from hospital admission to discharge. c, Immunofluorescence antibody staining, repeated twice in duplicate, for detection of IgG and IgM bound to SARS-CoV-2-infected Vero cells, assessed with plasma (diluted 1:20) obtained at days 7-9 and 20 following symptom onset. d-f, Frequency (left set of plots) of $C D 27^{\text {hi }} \mathrm{CD} 38^{\text {hi }} \mathrm{ASCs}$ (gated on $\mathrm{CD} 3^{-} \mathrm{CD} 19^{+}$lymphocytes) and activated ICOS ${ }^{+} \mathrm{PD}-1^{+} \mathrm{T}_{\mathrm{FH}}$ cells (gated on $\mathrm{CD} 4^{+} \mathrm{CXCR} 5^{+}$lymphocytes) (d), activated $\mathrm{CD} 38^{+} \mathrm{HLA}-\mathrm{DR}{ }^{+} \mathrm{CD} 8^{+}$or $\mathrm{CD} 4^{+} \mathrm{T}$ cells $(\mathbf{e})$, and CD $14^{+}$CD $16^{+}$monocytes and activated $\mathrm{HLA}^{-} \mathrm{DR}^{+}$natural killer (NK) cells (gated on CD3-CD14-CD56+ cells) (f), detected by flow cytometry of blood collected at days 7-9 and 20 following symptom onset in the patient and in healthy donors ( $n=5$; median with interquartile range); gating examples at right. Bottom right histograms and line graphs, staining of granzyme $A(G Z M A(A)$ ), granzyme $B(G Z M B(B)$ ), granzyme $K(G Z M K(K))$, granzyme $M(G Z M M(M))$ and perforin (Prf) in parent $C D 8^{+}$and $C D 4^{+}$ T cells and activated $\mathrm{CD} 38^{+} \mathrm{HLA}-\mathrm{DR}^{+} \mathrm{CD} 8^{+}$and $\mathrm{CD} 4^{+} \mathrm{T}$ cells. Gating and experimental details are in Extended Data Fig. 3.
H7N9 disease, which had elevated cytokines IL-6, IL-8, IL-10, MIP-1 $\beta$ and IFN- $\gamma^{6}$, minimal pro-inflammatory cytokines and chemokines were found in this patient with COVID-19, even while she was symptomatic at days 7-9.

As the single-nucleotide polymorphism rs $12252-\mathrm{C} / \mathrm{C}$ in the gene IFITM3 (which encodes interferon-induced transmembrane protein 3) is linked to severe influenza ${ }^{6,7}$, we analyzed IFITM3-rs12252 in the patient with COVID-19 and found the 'risk' IFITM3rs12252-C/C variant (Extended Data Fig. $2 \mathrm{~b})$. As the prevalence of IFITM3-rs12252$\mathrm{C} / \mathrm{C}$ in the Chinese population is $26.5 \%$ (the 1000 Genomes Project) ${ }^{6}$, further investigation of the IFITM3-rs12252-C/C allele in larger cohorts of people with COVID-19 is worth pursuing.

Collectively, our study provides novel contributions to the understanding of the breadth and kinetics of immune responses during a non-severe case of COVID-19. This patient did not experience complications of respiratory failure or acute respiratory distress syndrome, did not require supplemental oxygenation, and was discharged within a week of hospitalization, consistent with non-severe but symptomatic disease. We have provided evidence on the recruitment of immune cell populations (ASCs, $\mathrm{T}_{\mathrm{FH}}$ cells and activated $\mathrm{CD} 4^{+}$and $\mathrm{CD}^{+} \mathrm{T}$ cells), together with IgM and IgG SARS-CoV-2-binding antibodies, in the patient's blood before the resolution of symptoms. We propose that these immune parameters should be characterized in larger cohorts of people with COVID-19 with different disease severities to determine whether they could be used to predict disease outcome and evaluate new interventions that might minimize severity and/or to inform protective vaccine candidates. Furthermore, our study indicates that robust multi-factorial immune responses can be elicited to the newly emerged virus SARS-CoV-2 and, similar to the avian H7N9 disease ${ }^{8}$, early adaptive immune responses might correlate with better clinical outcomes.

\section{Reporting Summary}

Further information on research design is available in the Nature Research Reporting Summary linked to this article.

\section{Online content}

Any methods, additional references, Nature Research reporting summaries, source data, extended data, supplementary information, acknowledgements, peer review information; details of author contributions and competing interests; and statements of 
data and code availability are available at https://doi.org/10.1038/s41591-020-0819-2.

\section{Data availability}

The source data underlying Fig. 1a,c-f and Extended Data Fig. 2 are provided as Source Data. Raw FACS data are shown in the manuscript. FACS source files are available from the authors upon request.

Irani Thevarajan ${ }^{1,2,8}$, Thi H. O. Nguyen ${ }^{3,8}$, Marios Koutsakos ${ }^{3}$, Julian Druce ${ }^{4}$, Leon Caly ${ }^{4}$, Carolien E. van de Sandt ${ }^{3,5}$, Xiaoxiao Jia ${ }^{3}$, Suellen Nicholson ${ }^{4}$,

Mike Catton ${ }^{4}$, Benjamin Cowie ${ }^{1,2}$, Steven Y. C. Tong (D) 1,2,6, Sharon R. Lewin (D) 1,2,7 and Katherine Kedzierska (D) 3 四

${ }^{1}$ Victorian Infectious Diseases Service, The Royal Melbourne Hospital at the Peter Doherty Institute for Infection and Immunity, Melbourne, Australia. ${ }^{2}$ Doherty Department, The University of Melbourne at The Peter Doherty Institute for Infection and Immunity, Melbourne, Australia. ${ }^{3}$ Department of Microbiology and Immunology, The University of Melbourne, at the Peter Doherty Institute for Infection and Immunity, Parkville, Australia. ${ }^{4}$ Victorian Infectious Diseases Reference Laboratory, The Royal Melbourne Hospital at The Peter Doherty Institute for Infection and Immunity, Melbourne,
Australia. ${ }^{5}$ Department of Hematopoiesis, Sanquin Research and Landsteiner Laboratory, Amsterdam UMC, University of Amsterdam, Amsterdam, Netherlands. ${ }^{6}$ Menzies School of Health Research, Charles Darwin University, Darwin, Australia. ${ }^{7}$ Department of Infectious Diseases, Alfred Hospital and Monash University, Melbourne, Australia.

${ }^{8}$ These authors contributed equally: Irani Thevarajan, Thi H. O. Nguyen.

$\bigotimes_{\text {e-mail: kkedz@unimelb.edu.au }}$

Published online: 16 March 2020

https://doi.org/10.1038/s41591-020-0819-2

References

1. McElroy, A. K. et al. Proc. Natl Acad. Sci. USA 112, 4719-4724 (2015)

2. Ellebedy, A. H. et al. Nat. Immunol. 17, 1226-1234 (2016).

3. Koutsakos, M. et al. Sci. Transl. Med. 10, eaan8405 (2018).

4. Wang, Z. et al. Nat. Commun. 9, 824 (2018)

5. Xu, Z. et al. Lancet Respir. Med. https://doi.org/10.1016/S22132600(20)30076-X (2020).

6. Wang, Z. et al. Proc. Natl Acad. Sci. USA 111, 769-774 (2014)

7. Everitt, A. R. et al. Nature 484, 519-523 (2012).

8. Wang, Z. et al. Nat. Commun. 6, 6833 (2015).

\section{Acknowledgements}

We thank C. Simmons and all SETREP-ID investigators for their support; Australian Partnership for Preparedness Research for Infectious Disease Emergencies for funding; and L. Rowntree for technical assistance. This work was funded by an NHMRC Investigator Grant (1173871; K.K.). C.E.S. was supported by a Marie Skłodowska-Curie Fellowship (792532); K.K. was supported by an NHMRC Senior Research Fellowship (1102792); S.R.L. was supported by an NHMRC Practitioner Fellowship and an NHMRC Program Grant; S.Y.C.T. was supported by an NHMRC Career Development Fellowship (1145033); and X.J. was supported by a China Scholarship Council-UoM Scholarship. We acknowledge public health partners; the major funder of Victorian Infectious Diseases Reference Laboratory, the Victorian Department of Health and Human Services; and staff involved in patient care.

\section{Author contributions}

All authors formulated ideas, designed the study and experiments and reviewed the manuscript; T.H.O.N., M.K., J.D., L.C., C.E.S., X.J. and S.N. performed experiments; T.H.O.N., M.K., J.D., L.C. and S.N. analyzed experimental data; and I.T., T.H.O.N., B.C., S.Y.C.T., S.R.L. and K.K. wrote the manuscript.

Competing interests

S.R.L.'s institution received funding for investigatorinitiated grants from Gilead Sciences, Merck, Viiv Healthcare and Leidos; and honoraria for advisory boards and educational activities (Gilead Sciences, Merck, Viiv Healthcare and Abbvie).

\section{Additional information}

Supplementary information is available for this paper at https://doi.org/10.1038/s41591-020-0819-2. 


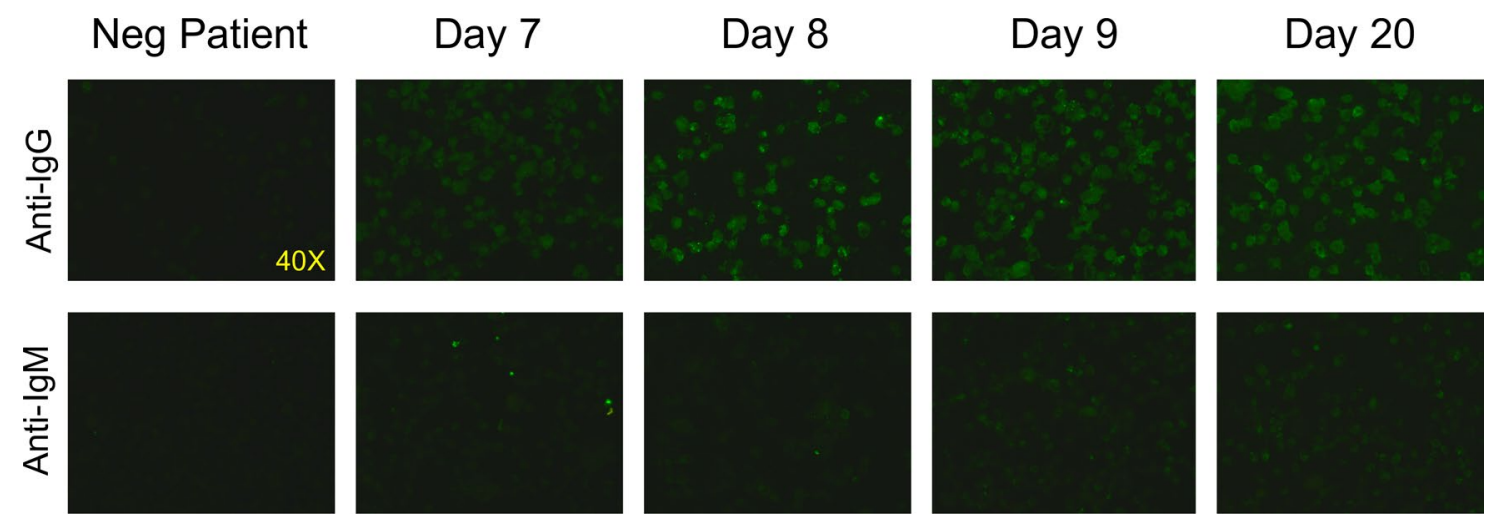

Extended Data Fig. 1 | Detection of IgG and IgM antibodies in SARS-CoV-2-infected Vero cells. Immunofluorescence antibody staining for the detection of IgG and IgM bound to SARS-CoV-2-infected vero cells using plasma (diluted 1:20) collected at d7-9 and d20 following onset of symptoms. Immunofluorescence antibody tests for the detection of IgG and IgM were performed using SARS-CoV-2-infected vero cells washed with PBS and methanol/acetone fixed onto glass slides. SARS-CoV-2 was isolated from another COVID patient, and infectivity at cell harvest was $\sim 80 \%$. 10 $\mu \mathrm{L}$ of a $1 / 20$ dilution of patient plasma in PBS from days 7, 8, 9 and 20 were incubated on separate wells for 30 mins at $37^{\circ} \mathrm{C}$, then washed in PBS and further incubated with $10 \mu \mathrm{L}$ of FITC-conjugated goat anti-human IgG and IgM (Euroimmun, Lübeck, Germany) before viewing on a EUROStar III Plus fluorescent microscope (Euroimmun). Prior to detection of IgM antibodies, samples were pre-treated with RF-SorboTech (Alere, Rüsselsheim, Germany) to remove IgG antibodies and rheumatoid factors, which may cause false-negative and false-positive IgM results, respectively. 
a
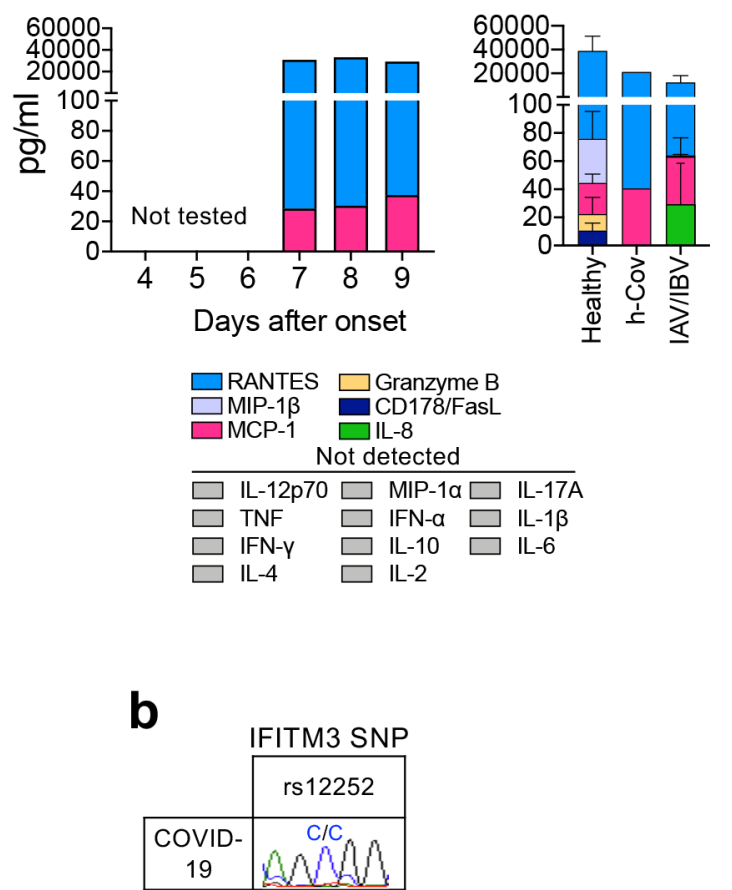

Extended Data Fig. 2 | Pro-inflammatory cytokines and chemokines and IFITM3-rs12252 in a patient with COVID-19. (a) Plasma levels of pro-inflammatory cytokines/chemokines in COVID-19 patient at d7-9, healthy individuals $(n=5$, mean \pm SEM), patient with HCoV-229e and influenza-infected patients ( $n=5$ ). Patient's plasma was diluted 1:4 before measuring cytokine levels (IL-2, IL-4, IL-6, IL-8, IL-10, IL-12p70, IL-17A, IL-1 $\beta$, IFN- $\alpha$, MIP-1 $\alpha$, MIP-1 $\beta$, MCP-1, CD178/FasL, granzyme B, RANTES, TNF, IFN- $\gamma$ ) using the Human CBA Kit (BD Biosciences, San Jose, California, USA). For RANTES, sera/plasma was also diluted to 1:50. Healthy donors D6-D10 were of a mean age of 32 (range 22-55 years; 40\% females). (b) 'risk' IFITM3-rs12252 genotyping for the COVID-19 patient. PCR was performed on genomic DNA extracted from patient's granulocytes (using QIAamp DNA Mini Kit, QIAGEN) to amplify the exon 1 rs12252 region using forward (5'-GGAAACTGTTGAGAAACCGAA-3') and reverse (5'-CATACGCACCTTCACGGAGT-3') primers. 
a

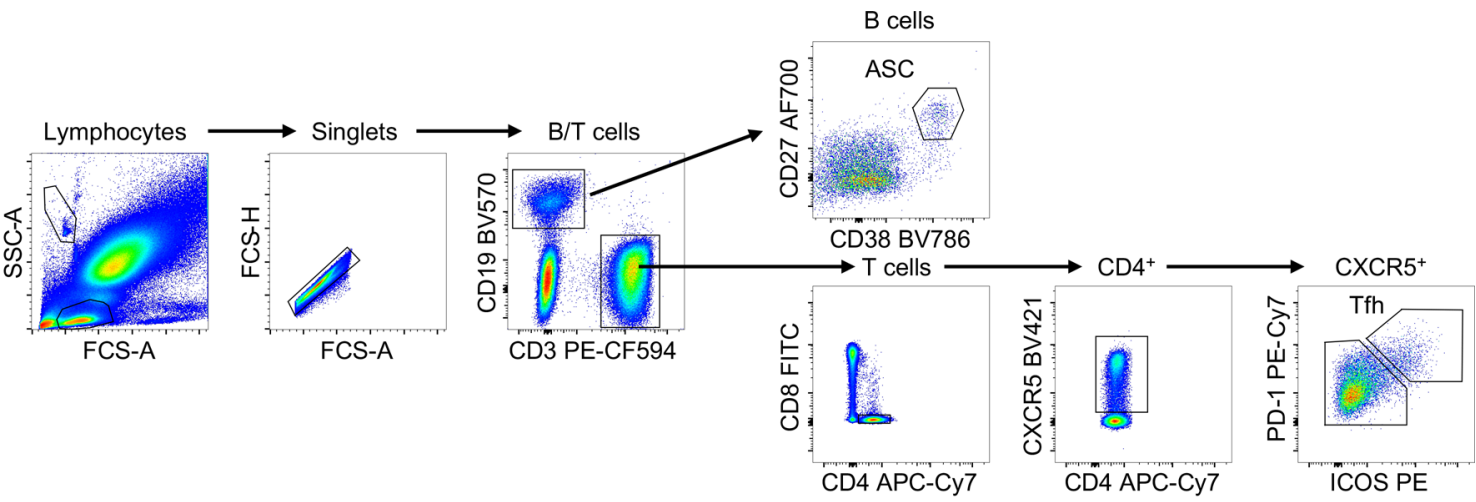

b
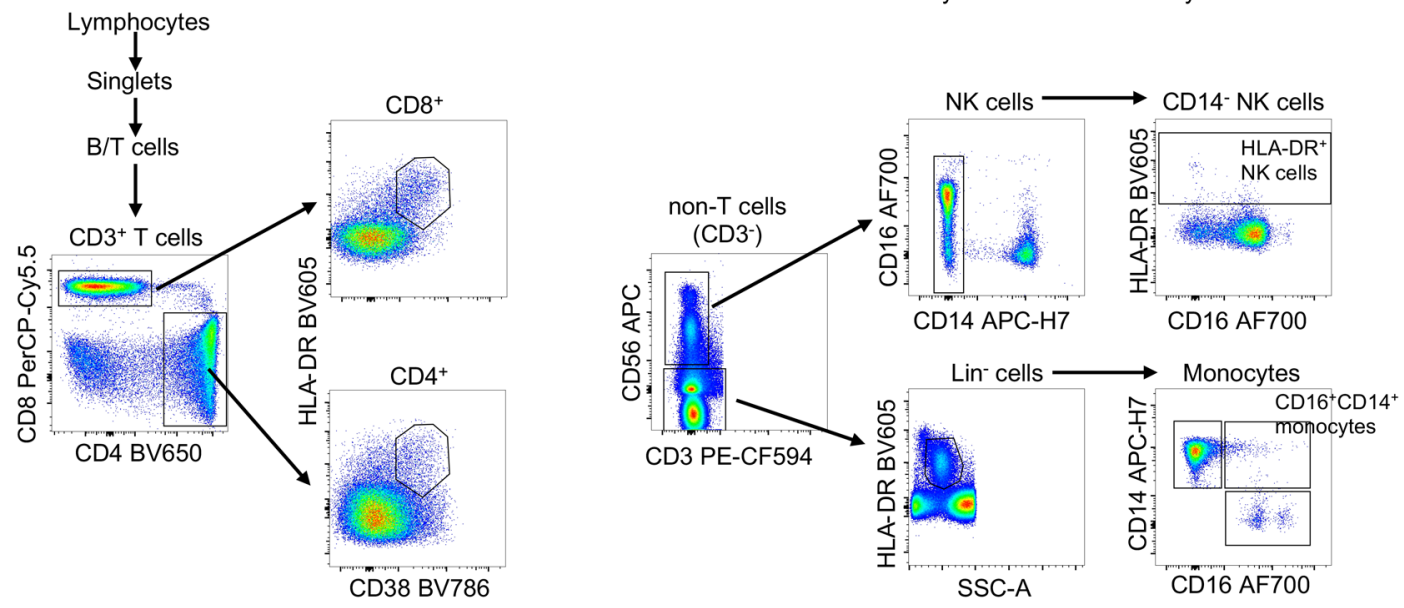

C
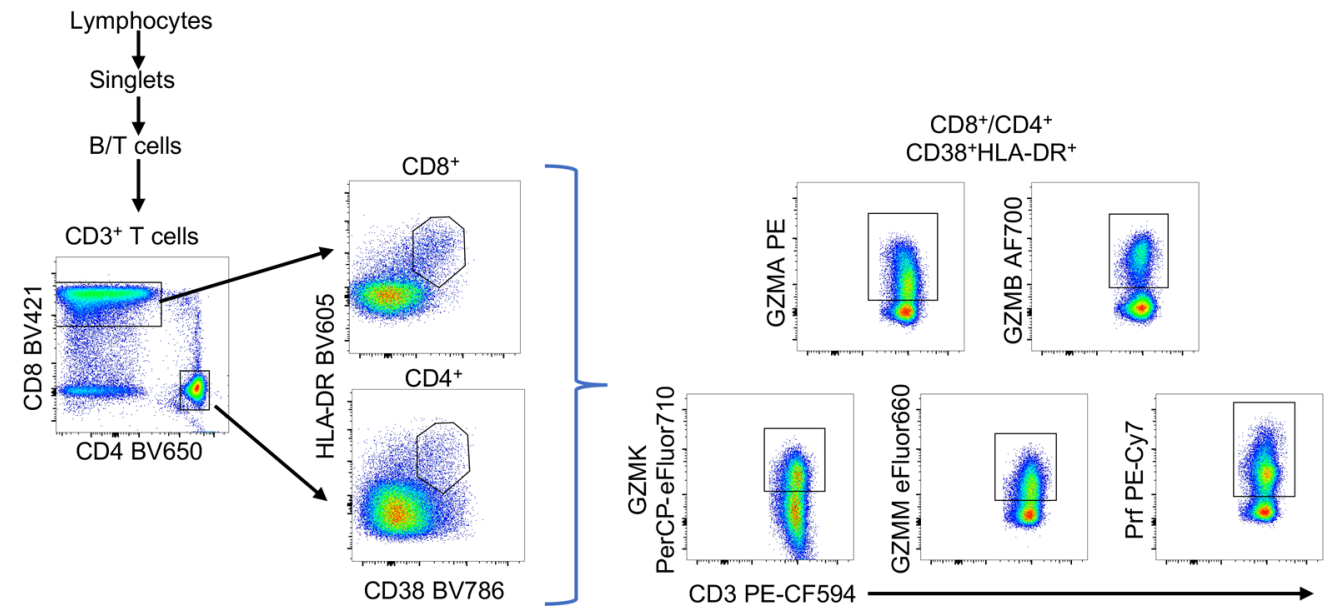

Extended Data Fig. 3 | Flow cytometry gating strategy for immune cell subsets. Gating panels are shown for (a) CD27 ${ }^{\text {hi }} C D 38^{\text {hi }}$ ASCs and activated ICOS+PD1+ Tfh cells; (b) activated CD38+HLA-DR ${ }^{+}$CD8 ${ }^{+}$and CD4 ${ }^{+}$T-cells, activated HLA-DR ${ }^{+} \mathrm{NK}$ cells and CD14+CD16 ${ }^{+}$monocytes; and (c) granzymes (GZM) A/B/K/M and perforin expression on CD8 ${ }^{+} / \mathrm{CD} 4^{+} \mathrm{T}$-cells and activated CD38+HLA-DR+ CD8 ${ }^{+} / \mathrm{CD}^{+}{ }^{+} \mathrm{T}$-cells. Fresh whole blood (200 $\mu$ l per stain) was used to measure $\mathrm{CD} 4{ }^{+} \mathrm{CXCR} 5^{+} \mathrm{ICOS}+\mathrm{PD} 1^{+}$follicular T cells (Tfh) and $\mathrm{CD} 3{ }^{-} \mathrm{CD} 19^{+} \mathrm{CD} 27^{\mathrm{hi}} \mathrm{CD} 38^{\text {hi }}$ antibody-secreting B cell (ASC; plasmablast) populations as described ${ }^{3}$ as well as activated $\mathrm{HLA}-\mathrm{DR}{ }^{+} \mathrm{CD} 38^{+} \mathrm{CD} 8^{+}$and $\mathrm{HLA}-\mathrm{DR}{ }^{+} \mathrm{CD} 38^{+} \mathrm{CD} 4^{+} \mathrm{T}$ cells, inflammatory $\mathrm{CD} 14^{+} \mathrm{CD} 16^{+}$and conventional $\mathrm{CD} 14+$ monocytes, activated $\mathrm{HLA}-\mathrm{DR}{ }^{+} \mathrm{CD} 3-\mathrm{CD} 56^{+} \mathrm{NK}$ cells. After the whole blood was stained for 20 mins at room temperature (RT) in the dark, samples were lysed with BD FACS Lysing solution, washed and fixed with 1\% PFA. Granzymes/perforin staining (patient d20) was performed using the eBioscience Foxp3/Transcription Factor Staining Buffer Set after the lysis step. All the samples were acquired on a LSRII Fortessa (BD). Flow cytometry data were analyzed using FlowJo v10 software. Healthy donors D1-D5 were of a mean age of 35 (range $24-42$ years, $40 \%$ females). 


\section{Reporting Summary}

Nature Research wishes to improve the reproducibility of the work that we publish. This form provides structure for consistency and transparency in reporting. For further information on Nature Research policies, see Authors \& Referees and the Editorial Policy Checklist.

\section{Statistics}

For all statistical analyses, confirm that the following items are present in the figure legend, table legend, main text, or Methods section.

$\mathrm{n} / \mathrm{a}$ | Confirmed

\ The exact sample size $(n)$ for each experimental group/condition, given as a discrete number and unit of measurement

$\square$ A statement on whether measurements were taken from distinct samples or whether the same sample was measured repeatedly

$\triangle$ The statistical test(s) used AND whether they are one- or two-sided

Xnly common tests should be described solely by name; describe more complex techniques in the Methods section.

$\bigotimes \square$ A description of all covariates tested

$\triangle \square$ A description of any assumptions or corrections, such as tests of normality and adjustment for multiple comparisons

A full description of the statistical parameters including central tendency (e.g. means) or other basic estimates (e.g. regression coefficient)

AND variation (e.g. standard deviation) or associated estimates of uncertainty (e.g. confidence intervals)

$\square$ For null hypothesis testing, the test statistic (e.g. $F, t, r$ ) with confidence intervals, effect sizes, degrees of freedom and $P$ value noted

$\triangle \square$ Give $P$ values as exact values whenever suitable.

\ $\square$ For Bayesian analysis, information on the choice of priors and Markov chain Monte Carlo settings

Х $\square$ For hierarchical and complex designs, identification of the appropriate level for tests and full reporting of outcomes

$\triangle \square$ Estimates of effect sizes (e.g. Cohen's $d$, Pearson's $r$ ), indicating how they were calculated

Our web collection on statistics for biologists contains articles on many of the points above.

\section{Software and code}

Policy information about availability of computer code

Data collection

BD FACS DIVA v8.0.1

Data analysis

FlowJo v10.5.3, Prism v8.3.1,

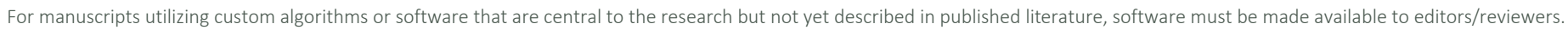
We strongly encourage code deposition in a community repository (e.g. GitHub). See the Nature Research guidelines for submitting code \& software for further information.

\section{Data}

Policy information about availability of data

All manuscripts must include a data availability statement. This statement should provide the following information, where applicable:

- Accession codes, unique identifiers, or web links for publicly available datasets

- A list of figures that have associated raw data

- A description of any restrictions on data availability

FACS plots, immunofluorescence and X-rays show raw data. Source data are supplied in "Source Data.xlsx" file

\section{Field-specific reporting}

Please select the one below that is the best fit for your research. If you are not sure, read the appropriate sections before making your selection.

$\bigotimes$ Life sciences $\quad \square$ Behavioural \& social sciences $\square$ Ecological, evolutionary \& environmental sciences

For a reference copy of the document with all sections, see nature.com/documents/nr-reporting-summary-flat.pdf 


\title{
Life sciences study design
}

All studies must disclose on these points even when the disclosure is negative.

Sample size The sample size was determined by the availability of samples: this is a COVID-2019 patient case report.

Data exclusions No data were excluded.

Replication Experiments in figure $1 \mathrm{c}$ were repeated twice in duplicates, with similar results. We have performed repeated flow cytometry stains and analyses, when feasible given the limited clinical material:

Staining for COVID patient ASC (Fig 1d) was repeated twice for d7 and d20, and three times for d8, d9 time-points, with similar results.

Staining for CD38+HLA-DR+CD8+ T cells (fig 1e) was repeated twice for d8 and d20, with similar results.

Due to a limitation in clinical specimens, the remaining stains for Tfhs, NK cells, CD38+HLA-DR+CD4+ T cells and monocytes was performed once.

Randomization It is a case study this randomization was not applicable

Blinding Experiments were not blinded as this was a Case Study

\section{Reporting for specific materials, systems and methods}

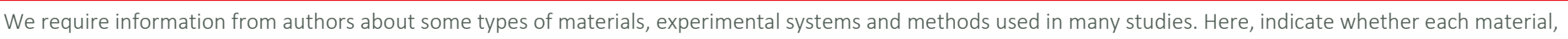

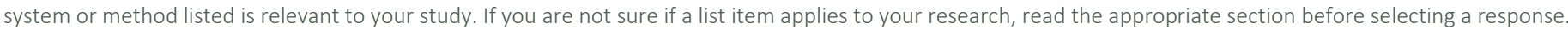

\begin{tabular}{l|l|l}
\multicolumn{2}{c}{ Materials \& experimental sy } \\
\hline $\mathrm{n} / \mathrm{a}$ & Involved in the study \\
\hline & $\bigotimes$ Antibodies \\
\hline & $\square$ Eukaryotic cell lines \\
\hline & $\square$ Animals and other organisms \\
$\square$ & $\square$ Human research participants \\
$\square$ Clinical data
\end{tabular}

Methods

n/a Involved in the study

Х ChIP-seq

$\square$ Х Flow cytometry

$\triangle \square$ MRI-based neuroimaging

\section{Antibodies}

Antibodies used

\author{
We used commercially-available antibodies: \\ Antibody/Clone/Fluorochrome/Dilution/Vendor/Cat number: \\ CD45RA HI100 FITC 1:50 BD Pharmingen 555488 \\ CD8a SK1 PerCP-Cy5.5 1:50 BD Pharmingen 565310 \\ CD56 MEM-188 APC 1:50 Biolegend 304610 \\ CD16 3 G8 AF700 1:50 Biolegend 302026 \\ CD14 MФP9 APC-Cy7 1:50 BD Pharmingen 560180 \\ CD19 HIB19 BV570 1:200 Biolegend 302236 \\ HLA-DR L243 BV605 1:50 Biolegend 307640 \\ CD4 SK3 BV650 1:200 BD Biosciences 563875 \\ CD27 L128 BV711 1:100 BD Horizon 563167 \\ CD38 HIT2 BV786 1:400 BD Horizon 563964 \\ CD3 UCHT1 PECF594 1:600 BD Biosciences 562280 \\ Granzymes/perforin panel: \\ Surface \\ CD45RA HI100 FITC 1:50 BD Pharmingen 555488 \\ CD8a RPA-T8 BV421 1:200 BD Pharmingen 301036 \\ CD14 MФP9 APC-H7 1:100 BD Pharmingen 560180 \\ CD19 SJ25C1 APC-H7 1:100 Biolegend 560177 \\ HLA-DR L243 BV605 1:50 Biolegend 307640 \\ CD4 SK3 BV650 1:200 BD Biosciences 563875 \\ CD27 L128 BV711 1:400 BD Horizon 563167 \\ CD38 HIT2 BV786 1:400 BD Horizon 563964 \\ CD3 UCHT1 PECF594 1:600 BD Biosciences 562280 \\ Intracellular \\ Granzyme A CB9 PE 1:50 eBioscience 12-9177-42
}




\section{Eukaryotic cell lines}

Policy information about cell lines

Cell line source(s)

Authentication

Mycoplasma contamination

Commonly misidentified lines (See ICLAC register)
Vero cells are African Green monkey kidney cells; were used for SARS-CoV-2 infections. Source ATCC - CRL01586 (Vero C1008 Kidney, Monkey). Lot 3338237

Cells line was not authenticated

Mycoplasma free (tested recently)

The cell line used is not listed in the ICLAC database

\section{Human research participants}

Policy information about studies involving human research participants

Population characteristics

Recruitment

Ethics oversight
This is a case report:

A 47-year old woman from Wuhan, Hubei province, China presented to an emergency department in Melbourne, Australia. Her symptoms commenced 4-days earlier with lethargy, sore throat, dry cough, pleuritic chest pain, mild dyspnoea and subjective fevers (Fig.1a). She travelled 11 days prior to presentation from Wuhan to Australia. She had no contact with the Huanan seafood market or known COVID-19 cases. She was otherwise healthy, non-smoker, taking no medications. Clinical examination revealed a temperature of $38.5 \mathrm{C}$, pulse rate 120 beats/minute, blood pressure 140/80 $\mathrm{mmHg}$, respiratory rate 22 breaths/ minute, and oxygen saturation $98 \%$, while breathing ambient air. Lung auscultation revealed bibasal rhonchi. At presentation day (d) 4, SARS-CoV-2 was detected by real-time reverse-transcriptase polymerase-chain-reaction (rRT-PCR) from a nasopharyngeal swab specimen. SARS-CoV-2 was again detected at d5-6 from nasopharyngeal, sputum and faecal samples, but undetectable from d7 (Fg.1a). Blood C-reactive protein was elevated at 83.2, with normal lymphocyte (4.3×109/L [range 4.0-12.0x109/L]) and neutrophil counts $(6.3 \times 109 / \mathrm{L}$ [range 2.0-8.0x109/L]). No other respiratory pathogens were detected. Her management was intravenous fluid rehydration without supplemental oxygenation. No antibiotics, steroids or antiviral agents were administered. Chest radiography demonstrated bibasal infiltrates at d5, which cleared on $\mathrm{d} 10$ (Fig.1b). She was discharged to home isolation on d11. Symptoms resolved completely by $\mathrm{d} 13$ and she remained well at $\mathrm{d} 20$.

Healthy donors ( $n=10), 4$ females, 6 males, mean age of 34 . Influenza-patients $(n=5), 1$ female, 4 males, mean age of 55. Human HCoV-229e Cov patient ( $n=1), 1$ male, age 27.

Healthy volunteer donors $>18$ years old were recruited through University of Melbourne (UoM) and virus-infected patients were recruited at Royal Melbourne Hospital (RMH). Signed informed consents were obtained from all blood donors prior to study.

Human experimental work was conducted according to the Declaration of Helsinki Principles and according to the Australian National Health and Medical Research Council Code of Practice. Participants provided written informed consent prior to the study. The study was approved by the Royal Melbourne Hospital (HREC Reference number: HREC/17/MH/53 and HREC/15/ MonH/64/2016.196) and University of Melbourne (ID \#1442952.1 and \#1443389.4) Human Research Ethics Committees.

Note that full information on the approval of the study protocol must also be provided in the manuscript.

\section{Flow Cytometry}

\section{Plots}

Confirm that:

\The axis labels state the marker and fluorochrome used (e.g. CD4-FITC).

\The axis scales are clearly visible. Include numbers along axes only for bottom left plot of group (a 'group' is an analysis of identical markers).

$\bigotimes$ All plots are contour plots with outliers or pseudocolor plots.

$\bigotimes$ A numerical value for number of cells or percentage (with statistics) is provided. 
Methodology

Sample preparation

Instrument

Software

Cell population abundance

Gating strategy
Whole blood staining was performed as outlined in fig legends to Extended Data

BD LSR Fortessa was used for acquisition of data

BD FACS Diva v8.0.1, Flow Jo v10.5.3

We have not sorted the PBMCs

Gating strategy has been described in methods, figure legends and as Extended Data figure 3

$\bigotimes$ Tick this box to confirm that a figure exemplifying the gating strategy is provided in the Supplementary Information. 